\title{
Benign Idiopathic Cystic Chondromalacia Of Auricle
}

\author{
Jeong Hwan Choi
}

\begin{abstract}
Benign idiopathic cystic chondromalacia (BICC) is an uncommon, benign, painless, and usually unilateral, dome-shaped cystic swelling in the helix or antihelix of the auricle. Common sites include the cymba concha, scaphoid fossa and triangular fossa. BICC can also be found in the literature reports labeled as auricular pseudocyst, endochondral pseudocyst, intracartilaginous cyst, and (pseudo) auricular seroma. Because BICC is fairly unknown, they can be misdiagnosed and as a consequence inappropriately treated. A 60-year-old man presented with a mass on the right auricle of 5-year duration. It had increased slightly in size over this period. He denied any history of auricular trauma. Physical examination revealed a firm $1.5 \times 1 \mathrm{~cm}$ sized firm mass in the scaphoid fossa and protruded slightly into the posterior helix. The patient underwent an excisional biopsy for diagnosis. The pathologic features of the mass were diagnostic for a BICC. Because BICC is fairly unknown, they can be misdiagnosed and as a consequence inappropriately treated.
\end{abstract}

Keywords: Cartilage disease; Ear cartilage; Treatment outcome

\section{Introduction}

Benign idiopathic cystic chondromalacia (BICC) is an uncommon, benign, painless, and usually unilateral, dome-shaped cystic swelling in the helix or antihelix of the auricle. Common sites include the cymba concha, scaphoid fossa and triangular fossa. BICC can also be found in the literature reports labeled as auricular pseudocyst, endochondral pseudocyst, intracartilaginous cyst, and (pseudo) auricular seroma.

Because BICC is fairly unknown, they can be misdiagnosed and as a consequence inappropriately treated. It was first described in English literature as pseudocyst by Engel in 1966 [1].

\section{Case Report}

A 60-year-old man presented with a mass on the right auricle

Manuscript submitted October 20, 2018, accepted October 29, 2018

Department of Otorhinolaryngology-Head and Neck Surgery, Inje University Sanggye Paik Hospital, Dong il-ro 1342, Nowon-gu, Seoul 01757, South Korea.Email: choijh92@paik.ac.kr

doi: https://doi.org/10.14740/jmc3193 of 5-year duration. It had increased slightly in size over this period. He denied any history of auricular trauma. Physical examination revealed a firm $1.5 \times 1 \mathrm{~cm}$ sized firm mass in the scaphoid fossa and protruded slightly into the posterior helix (Fig. 1). The patient underwent an excisional biopsy for diagnosis. After local infiltration with $2 \%$ lidocaine and 1:100,000 epinephrine, a helical incision was made. After elevation of the subperichondrial flap beyond the mass, a tangential incision was made around mass through the cartilage. After extirpating the mass, the perichondrial flap was returned to its original position and sutured back. In order to prevent hematoma formation, a compression dressing was applied for 2 days after surgery. Histological examination revealed an intracartilaginous cyst without an epithelial lining, and fibrosis with focal destruction and inflammation of the cartilage (Fig. 2). The pathologic features of the mass were diagnostic for a BICC. The patient is now 7 months post surgery and is free of recurrence and cosmetic deformity of his auricle.

\section{Discussion}

Although the etiology of BICC is largely unknown, an inflammatory response to repetitive trauma is thought to be responsible [2]. This however, was not present in our patient's history. The second hypothesis is that the auricular cartilage dysplasia can be a cause of BICC [3]. In the embryologic development of auricle from the first and second branchial arch, residual tissue may be leaved within the cartilage by dysplasia. An additional stimulus might be required to open this potential space to form a BICC [4].

Histologically, BICC is an intracartilaginous cystic space without epithelial lining (Figs. 2, 3A) unlike other auricular pathology such as otohematoma (Fig. 3B), hence, called pseudocyst [5]. The space is lined with mild inflammatory cells and granulation cell.

The differential diagnosis of BICC include benign cutaneous cysts such as epidermal, trichilemmal and dermoid cysts; inflammatory lesions such as cellulitis, relapsing polychondritis and chondrodermatitis nodularis helicis gouty tophus; and even tumors such as adnexal cutaneous tumors, chondromas, fibromas, angiomas, and even angiosarcoma [6].

Various treatment options are reported in the literature without a clear consensus and various results. The therapeutic goal is to remove the BICC with restored the normal architecture of the auricle and without recurrence. Treatment options include intralesional injection of corticosteroids after aspiration and surgical excision. A simple needle aspiration often 


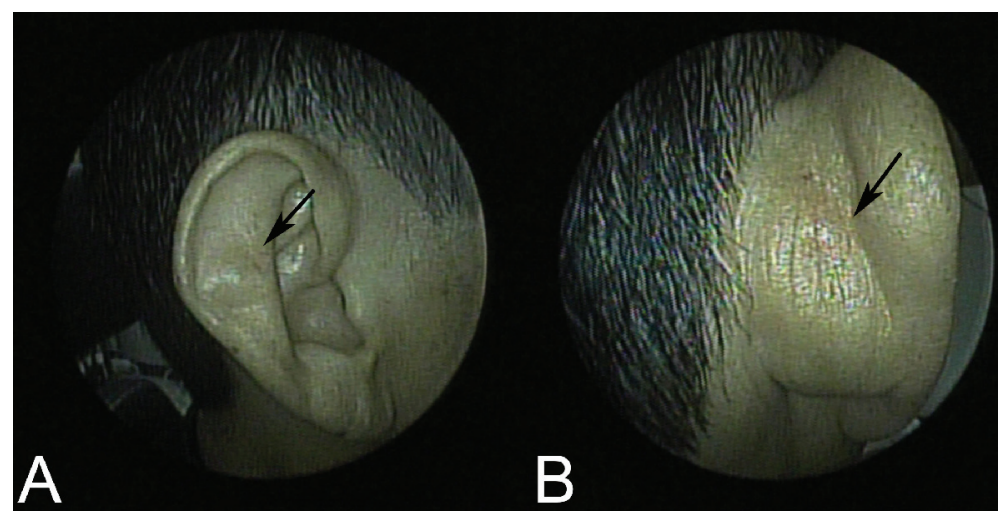

Figure 1. (A) A painless, dome-shaped cystic mass (arrow) in the right auricle. (B) The cystic mass protrudes slightly into the posterior side of auricle.

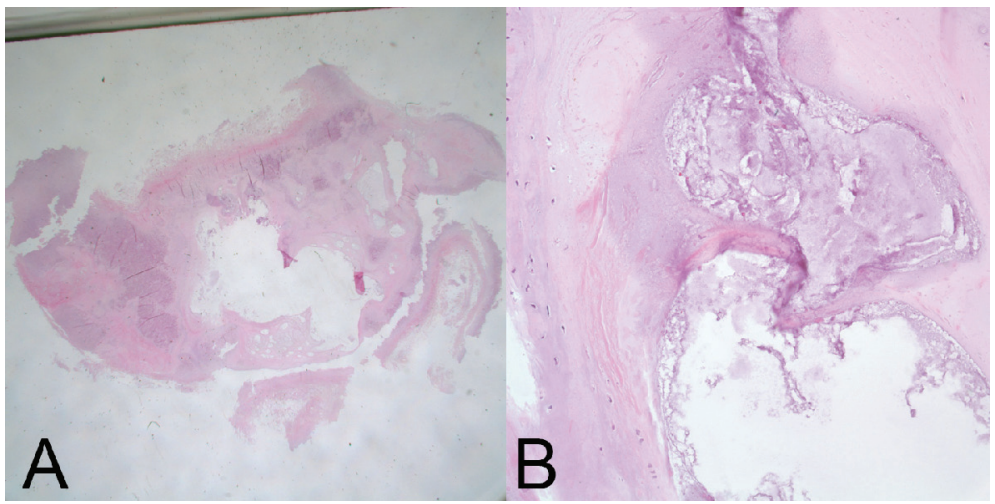

Figure 2. (A) Low power examination of the lesion reveals intracartilaginous cavity. (B) On the high power magnification, there is no epithelial lining of the cyst. Instead, inflammatory cells and granulation tissue can be seen lining the cyst (H\&E staining).

causes fluid to reaccumulate and recurrence. In "de-roofing" techniques [2], the anterior wall of a BICC is resected along the entire circumference of the lesion. The granulation on the posterior wall is also curetted, leaving the posterior wall intact. By leaving the posterior wall intact, there is no cosmetic deformity. Even though our patient healed without any cosmetic deformity, we wound recommend de-roofing the anterior wall of the cyst instead of total excision.

Because BICC is fairly unknown, they can be misdiagnosed and as a consequence inappropriately treated. We also wound recommend de-roofing the anterior wall of the cyst instead of total excision.

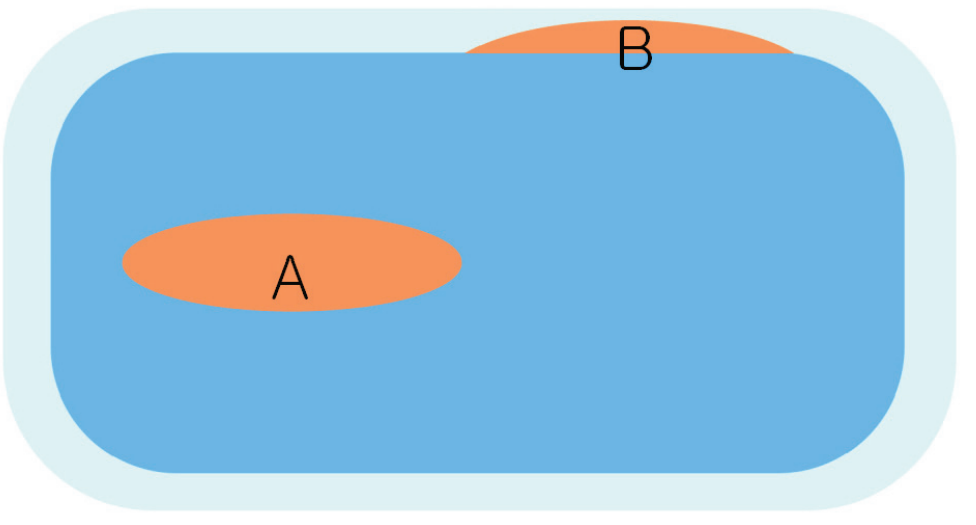

Figure 3. Benign idiopathic cystic chondromalacia (A, orange) is an intracartilaginous cystic space in contrast to other auricular conditions such as otohematoma ( $\mathrm{B}$, orange) is located between perichondrium (light sky blue) and cartilage (sky blue). Here you would describe each figure and its significance. 


\section{Conflict of Interest}

The author had no conflict of interest on this study.

\section{Financial Support}

There is no financial and material support or any funding to this study.

\section{References}

1. Engel D. Pseudocysts of the auricle in Chinese. Arch Oto- laryngol. 1966;83(3):197-202.

2. Choi S, Lam KH, Chan KW, Ghadially FN, Ng AS. Endochondral pseudocyst of the auricle in Chinese. Arch Otolaryngol. 1984;110(12):792-796.

3. Ichioka S, Yamada A, Ueda K, Harii K. Pseudocyst of the auricle: case reports and its biochemical characteristics. Ann Plast Surg. 1993;31(5):471-474.

4. Lee JA, Panarese A. Endochondral pseudocyst of the auricle. J Clin Pathol. 1994;47(10):961-963.

5. Lim CM, Goh YH, Chao SS, Lynne L. Pseudocyst of the auricle. Laryngoscope. 2002;112(11):2033-2036.

6. Salgado CJ, Hardy JE, Mardini S, Dockery JM, Matthews MS. Treatment of auricular pseudocyst with aspiration and local pressure. J Plast Reconstr Aesthet Surg. 2006;59(12):1450-1452. 